\title{
Two-Dimensional Functionalized Germananes as
}

\section{Photoelectrocatalysts}

Siowwoon Ng, ${ }^{1}$ Jiri Sturala, ${ }^{2}$ Jan Vyskocil, ${ }^{3}$ Petr Lazar, ${ }^{4}$ Jana Martincova, ${ }^{5}$ Jan Plutnar, ${ }^{3}$ Martin Pumera $^{1,3,6,7,8^{*}}$

${ }^{1}$ Future Energy and Innovation Laboratory, Central European Institute of Technology, Brno University of Technology, Purkyňova 123, 61200 Brno, Czech Republic

${ }^{2}$ Department of Inorganic Chemistry, Faculty of Chemical Technology, University of Chemistry and Technology Prague, Technická 5, Prague 16628, Czech Republic

${ }^{3}$ Center for Advanced Functional Nanorobots, Department of Inorganic Chemistry, Faculty of Chemical Technology, University of Chemistry and Technology Prague, Technická 5, Prague 16628, Czech Republic

${ }^{4}$ Regional Centre of Advanced Technologies and Materials, Palacký University Olomouc, 17. listopadu 1192/12, 77146 Olomouc, Czech Republic

${ }^{5}$ Department of Physical Chemistry, Faculty of Science, Palacký University Olomouc, 17. listopadu 1192/12, 77146 Olomouc, Czech Republic 
${ }^{6} 3 \mathrm{D}$ Printing \& Innovation Hub, Department of Food Technology, Mendel University in Brno, Zemedelska 1, 61300 Brno, Czech Republic

${ }^{7}$ Department of Chemical and Biomolecular Engineering, Yonsei University, 50 Yonsei-ro, Seodaemun-gu, Seoul 03722, South Korea

${ }^{8}$ Department of Medical Research, China Medical University Hospital, China Medical University, No. 91 Hsueh-Shih Road, Taichung 40402, Taiwan

*Corresponding author: pumera.research@gmail.com 


\section{Experimental - Materials characterizations}

The surface morphology of the germananes was imaged by scanning electron microscope (FEI Verios 460L, USA).

Fourier-transform infrared (FTIR) measurements were performed with an iS50R FTIR spectrometer (Thermo Scientific, USA) in the range of 4000-400 $\mathrm{cm}^{-1}$. A KBr beam splitter with a resolution of $4 \mathrm{~cm}^{-1}$ was employed for the measurements. The samples were measured in a $\mathrm{KBr}$ pellet made of finely grounded $\mathrm{KBr}(200 \mathrm{mg})$ and the sample $(1 \mathrm{mg})$.

X-ray diffraction (XRD) measurements were performed using a Bruker D8 Discoverer (Bruker, Germany) diffractometer with a $\mathrm{Cu} \mathrm{K} \alpha$ radiation source $(\lambda=1.540598 \AA)$ and a para-focusing Bragg-Brentano geometry. The data were collected over an angular range of $5-90^{\circ}(2 \theta)$ with a counting time of 0.5 s per step and a step size of $0.0167^{\circ}(2 \theta)$. HighScore Plus software was used for data processing.

Thermogravimetric analysis was carried out using a Themys TGA (SETARAM instrument) linked to a mass spectrometer (OMNI star) at a temperature range between 30 and $600{ }^{\circ} \mathrm{C}$ and a heating rate of $10^{\circ} \mathrm{C} \mathrm{min}^{-1}$. The measurements were performed with helium as carrier gas with a flow rate of $100 \mathrm{~mL} \mathrm{~min}^{-1}$.

For Raman and micro-photoluminescence (PL) measurement, the powdered sample was deposited onto a metal plate. Raman spectroscopic measurements were performed with an inVia Raman microscope (Renishaw, England) coupled with a CCD detector containing backscattering geometry and a $20 \times$ magnification objective lens. The measurements were carried out using a DPSS Nd-YAG laser $(532 \mathrm{~nm}, 50 \mathrm{~mW})$ with an applied power of $0.05 \%$. The peaks in the spectra

were referenced to a laser peak at $0 \mathrm{~cm}^{-1}$. The micro-PL spectra were measured by the mentioned 
Raman confocal microscope. The PL spectra were measured using an Nd-YAG laser (532 nm, 50 $\mathrm{mW}$ ) and an applied power of $0.0001 \%$ (except for sample Ge-H, $0.1 \%$ was used). The spectra were collected with a $20 \times$ objective and a TE-cooled CCD camera.

The macro-PL measurements were performed using Fluorolog Extreme system (Horiba, France) equipped with a Xe lamp $(450 \mathrm{~W})$ and double excitation monochromator. The emission spectra were measured by monochromator iHR320 with a thermoelectrically cooled PMT detector.

X-ray photoelectron spectroscopy (XPS) measurement was performed using high-resolution XPS SPECS spectrometer equipped with an XR 50 MF monochromatic X-ray radiation source $(1486.7 \mathrm{eV})$ and a Phoibos 150 2D CCD hemispherical analyzer and detector. The pressure inside the chamber during the measurements was $5 \times 10^{-10}$ mbar or lower. Wide-scan surveys were performed with $E_{\mathrm{p}}=80 \mathrm{eV}$, with subsequent high-resolution scans of the desired core lines with $E_{\mathrm{p}}=50 \mathrm{eV}$. The samples were placed on double-sided carbon tape. The binding energy values are referenced to the adventitious carbon peak at $284.8 \mathrm{eV}$. An electron gun was used to eliminate the charging effect of samples during the measurement.

Optical absorbance was measured by PerkinElmer Lambda 850+ spectrometer. The samples were placed inside an integrating sphere coupled to a PMT detector. Tungsten lamp (350-850 nm) was used as the irradiation source and $0.16 \mathrm{~s} \mathrm{~nm}^{-1}$ integration time was used for the measurements. Quartz glass cuvette with the path length of $1 \mathrm{~cm}$ was filled with aqueous suspensions of germananes $\left(1 \mathrm{mg} \mathrm{mL}^{-1}\right)$ and the samples were measured immediately. 


\section{Additional Materials Characterizations}
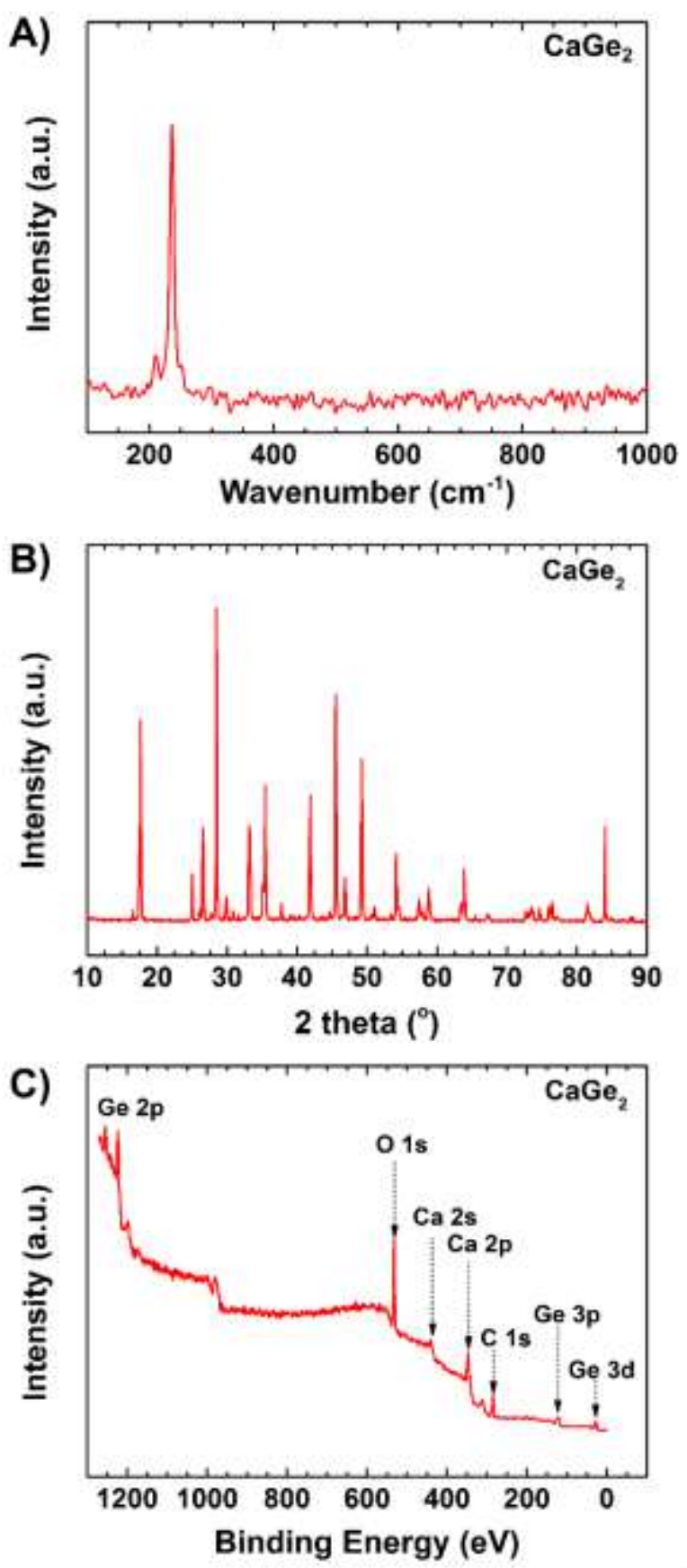

Figure S1. Material characterizations for $\mathrm{CaGe}_{2}$ : (A) Raman spectrum, (B) XRD pattern, and (C) XPS survey spectrum. 

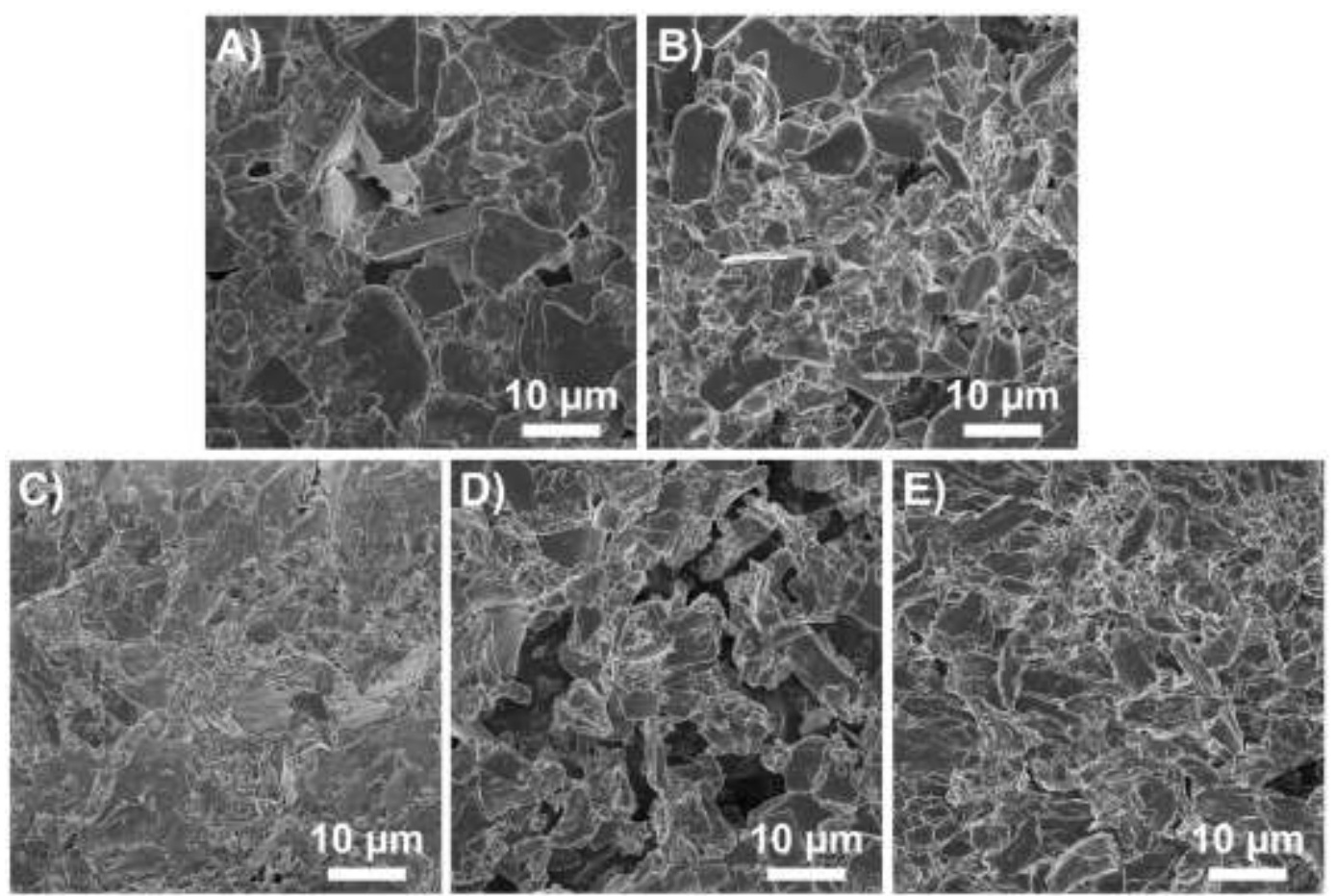

Figure S2. SEM images in low magnification for an overview of (A) germanane, Ge-H, (B) methyl germanane, Ge-Me, (C) propyl germanane, Ge-Pr, (D) hydroxypropyl germanane, Ge-PrOH, and (E) 2-(methoxycarbonyl)ethyl germanane, Ge-PE. 

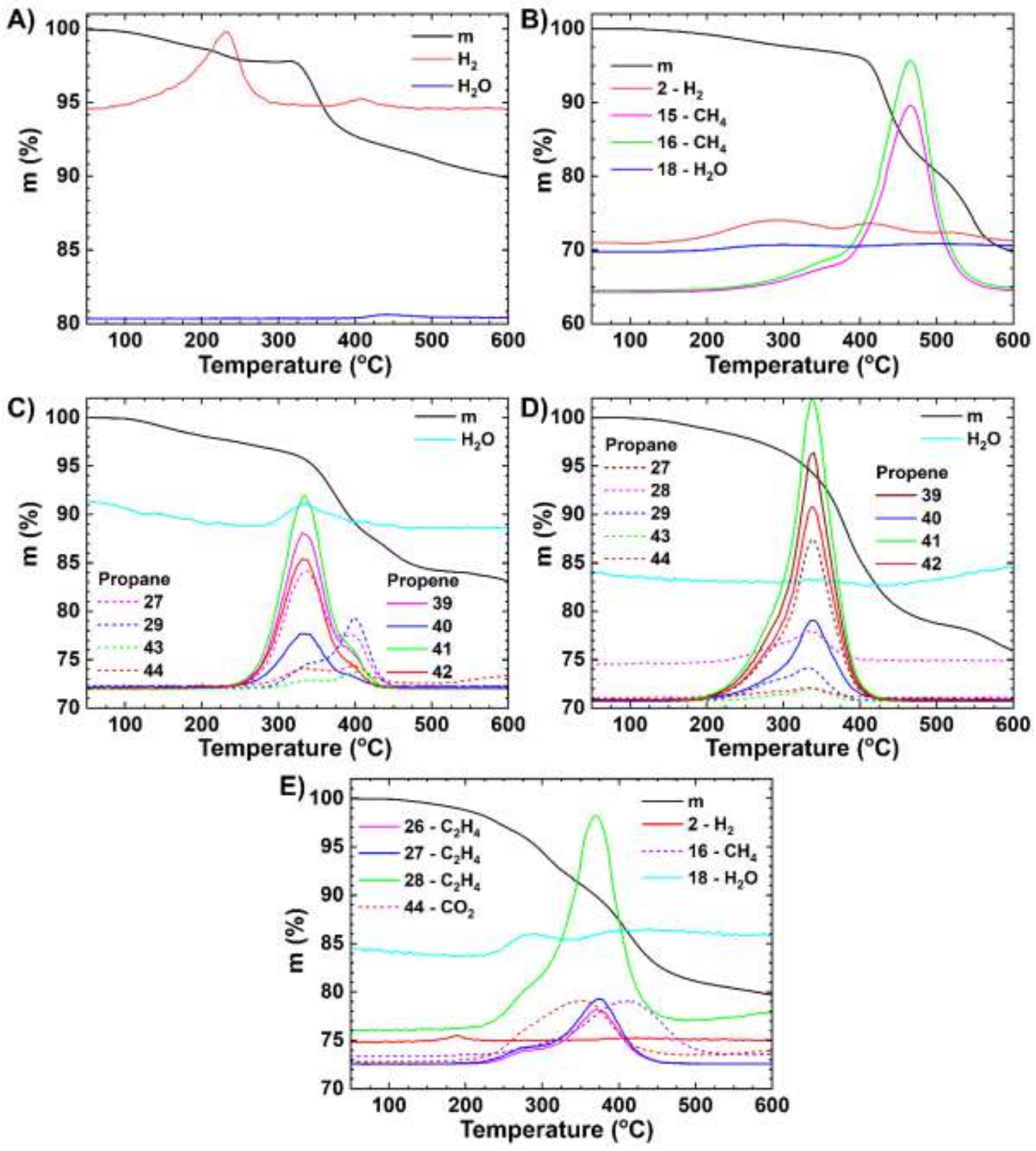

Figure S3. Mass spectroscopy (MS) analysis of evolved gases for all germananes under heating and under an inert atmosphere: (A) Ge-H, (B) Ge-Me, (C) Ge-Pr, (D) Ge-PrOH, and (E) Ge-PE. 
A) $\mathrm{Ge} 2 \mathrm{p}$

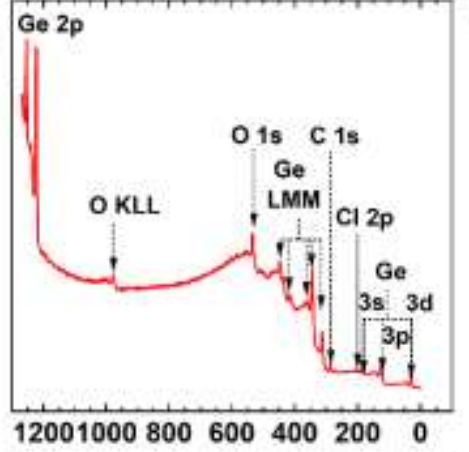

C) $\mathrm{Ge} 2 \mathrm{p}$

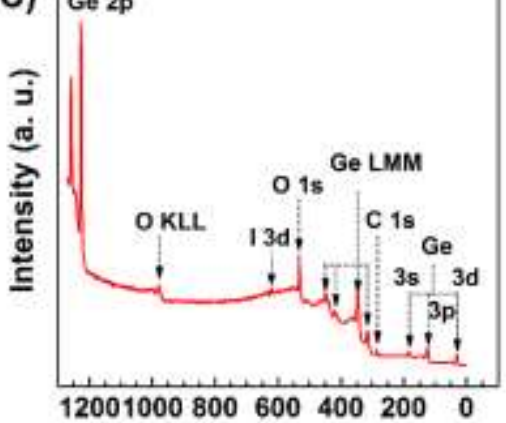

E)

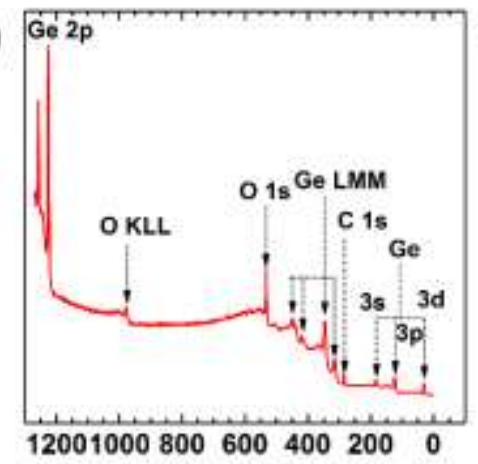

B)

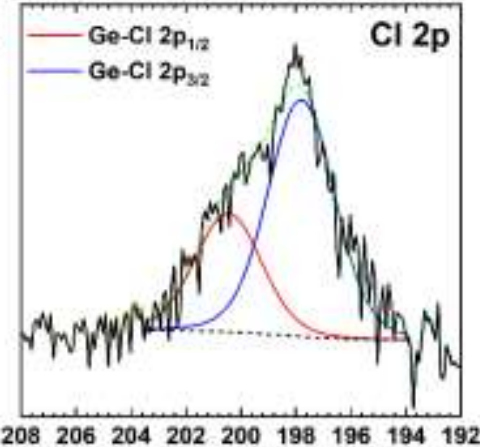

$208206204202200198 \quad 196 \quad 194 \quad 192$

D)

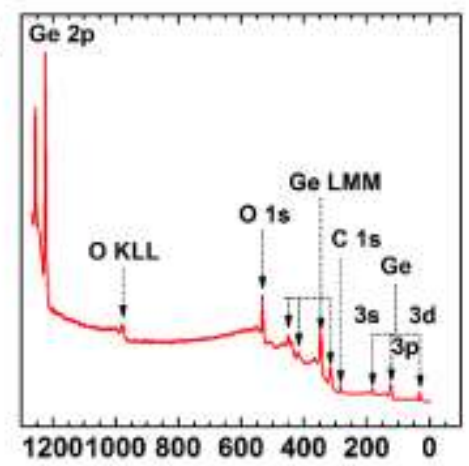

F) Ge $2 p$

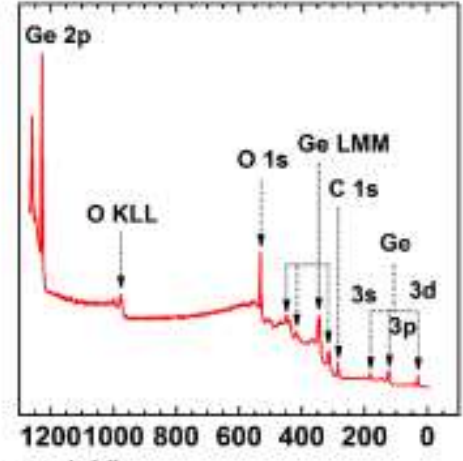

Binding energy (eV)

Figure S4. XPS survey spectra for all germananes (A) Ge-H, (C) Ge-Me, (D) Ge-Pr, (E) Ge-PrOH, and (F) Ge-PE, and (B) high-resolution $\mathrm{Cl} 2 \mathrm{p}$ spectrum for Ge-H. 
Table S1. Elemental analysis based on XPS measurement for all germananes.

\begin{tabular}{|c|c|c|c|c|c|c|c|c|c|}
\hline \multirow[t]{2}{*}{ Germanane } & & \multicolumn{2}{|c|}{ Ge } & \multicolumn{3}{|c|}{$\mathbf{C}$} & \multicolumn{2}{|c|}{$\mathbf{O}$} & \multirow{2}{*}{$\begin{array}{c}\mathrm{X} \\
\mathrm{Ge}-\mathrm{X}\end{array}$} \\
\hline & & Ge-O & Ge-R & $\mathbf{C}=\mathbf{O}$ & $\begin{array}{l}\text { C-C + } \\
\text { adv. C }\end{array}$ & Ge-C & $\begin{array}{l}\text { C-O + } \\
\text { adv. O }\end{array}$ & Ge-O & \\
\hline \multirow{3}{*}{$\begin{array}{l}\text { Ge-H } \\
(X=C l)\end{array}$} & Total at $\%$ & \multicolumn{2}{|c|}{45.4} & \multicolumn{3}{|c|}{24.4} & \multicolumn{2}{|c|}{26.8} & 3.4 \\
\hline & Individual at $\%$ & 5.0 & 95.0 & \multirow[t]{2}{*}{ N/A } & 100.0 & \multirow[t]{2}{*}{ N/A } & 92.9 & 7.2 & 100.0 \\
\hline & No. of atoms & 2.3 & 43.1 & & 24.4 & & 24.9 & 1.9 & 3.4 \\
\hline \multirow{3}{*}{$\begin{array}{l}\text { Ge-Me } \\
(X=I)\end{array}$} & Total at $\%$ & \multicolumn{2}{|c|}{23.6} & \multicolumn{3}{|c|}{42.0} & \multicolumn{2}{|c|}{34.3} & 0.2 \\
\hline & Individual at $\%$ & 3.3 & 96.7 & \multirow[t]{2}{*}{ N/A } & 60.7 & 39.3 & 92.5 & 7.5 & 100.0 \\
\hline & No. of atoms & 0.8 & 22.8 & & 25.5 & 16.5 & 31.7 & 2.6 & 0.2 \\
\hline \multirow{3}{*}{$\begin{array}{l}\text { Ge-Pr } \\
(\mathrm{X}=\mathrm{Br})\end{array}$} & Total at $\%$ & \multicolumn{2}{|c|}{28.5} & \multicolumn{3}{|c|}{32.4} & \multicolumn{2}{|c|}{39.1} & \multirow[t]{3}{*}{$0^{*}$} \\
\hline & Individual at $\%$ & 5.8 & 94.2 & N/A & 75.6 & 24.4 & 88.3 & 11.7 & \\
\hline & No. of atoms & 1.7 & 26.9 & & 24.4 & 7.9 & 34.5 & 4.6 & \\
\hline \multirow{3}{*}{$\begin{array}{l}\text { Ge-PrOH } \\
(\mathrm{X}=\mathrm{Br})\end{array}$} & Total at \% & \multicolumn{2}{|c|}{32.0} & \multicolumn{3}{|c|}{32.3} & \multicolumn{2}{|c|}{35.8} & \multirow[t]{3}{*}{$0^{*}$} \\
\hline & Individual at $\%$ & 9.9 & 90.2 & N/A & 72.8 & 27.2 & 86.7 & 13.3 & \\
\hline & No. of atoms & 3.1 & 28.8 & & 23.5 & 8.8 & 31.0 & 4.7 & \\
\hline \multirow{3}{*}{$\begin{array}{l}\text { Ge-PE } \\
(\mathrm{X}=\mathrm{Br})\end{array}$} & Total at $\%$ & \multicolumn{2}{|c|}{20.6} & \multicolumn{3}{|c|}{46.0} & \multicolumn{2}{|c|}{33.5} & \multirow[t]{3}{*}{$0^{*}$} \\
\hline & Individual at $\%$ & 7.1 & 92.9 & 13.8 & 74.9 & 11.3 & 91.7 & 8.3 & \\
\hline & No. of atoms & 1.5 & 19.1 & 6.3 & 34.4 & 5.2 & 30.7 & 2.8 & \\
\hline
\end{tabular}

Ge-O: Ge-oxide(s), i.e. Ge-O, Ge-OH

adv.: adventitious

N/A: not available

$0^{*}$ : not detected

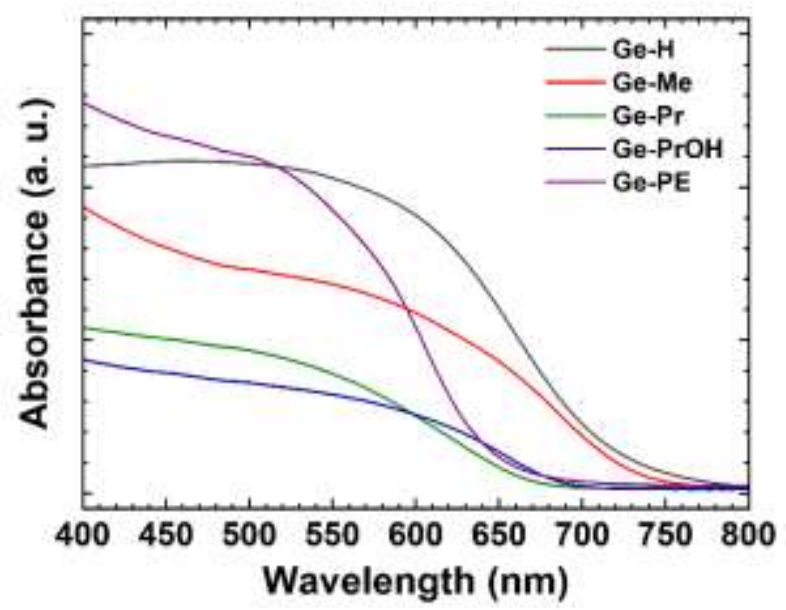

Figure S5. Absorbance spectra of all germananes, Ge-H, Ge-Me, Ge-Pr, Ge-PrOH, and Ge-PE, in the visible spectral region. 


\section{Additional Discussions}

The XPS analyses revealed trace amount of $\mathrm{Cl}$ in $\mathrm{Ge}-\mathrm{H}$ and $\mathrm{O}$ in all germananes. For $\mathrm{Cl}$, we performed the formation energy calculation with the $\mathrm{Cl}$ atom bound i) to one of the $\mathrm{H}$ atoms on the surface of Ge-H (adsorbed Cl, Figure S6A), and ii) directly to one of the Ge atoms by the formal substitution of attached $\mathrm{H}$ (substituted $\mathrm{Cl}$, Figure S6B) to estimate the thermodynamic stability of chlorine impurity defects. Taking the chemical potential of $\mathrm{Cl}$ as the reference state, we obtained positive formation energy of $0.97 \mathrm{eV}$ for the adsorption of $\mathrm{Cl}$ and negative energy of $-0.51 \mathrm{eV}$ for the substitution of $\mathrm{Cl}$. Thus, the substitution of hydrogen by chlorine is thermodynamically favorable, which explains the presence of trace $\mathrm{Cl}$ in $\mathrm{Ge}-\mathrm{H}$.

Further, we separately calculated the band gap of the Ge-H structures with the substituted and adsorbed $\mathrm{Cl}$. In either case, the band gap is reduced merely by $0.02 \mathrm{eV}$, so it remains similar to that of an ideal Ge-H. Thus, the results corroborate that the $\mathrm{Cl}$ impurities do not alter the photoadsorption of Ge-H. The influence of $\mathrm{O}$ impurities is more complicated to evaluate because $\mathrm{O}$ can be present in various chemical forms. In addition, it is very challenging to track and accurately quantify the oxygen concentration in the samples due to the environmental contamination (adventitious $\mathrm{C}$ and $\mathrm{O}$ ). Based on our synthetic protocol, the most probable form of oxygen is the adsorbed $-\mathrm{OH}$. From the FTIR spectrum of Ge-H in Figure 2B, the absence of the broad band at $800-1000 \mathrm{~cm}^{-1}$ (only a small peak at $\approx 830 \mathrm{~cm}^{-1}$ ) that is associated with various germanium oxides implies their minimal presence within Ge-H. ${ }^{1,2}$ The total energy of $\mathrm{O}$ adsorbed on top of a $\mathrm{H}$ atom in $\mathrm{Ge}-\mathrm{H}$ is very similar (with the energy difference of $0.06 \mathrm{eV}$ ) to the $-\mathrm{OH}$ directly bonded to a Ge atom, shown in Figure S6C. The band gap again remains the same, but the adsorbed -OH leads to the emergence of a localized mid-gap state above the Fermi level. These findings corroborate the negligible influence of impurities to the photo-activities shown in Figure 6. 

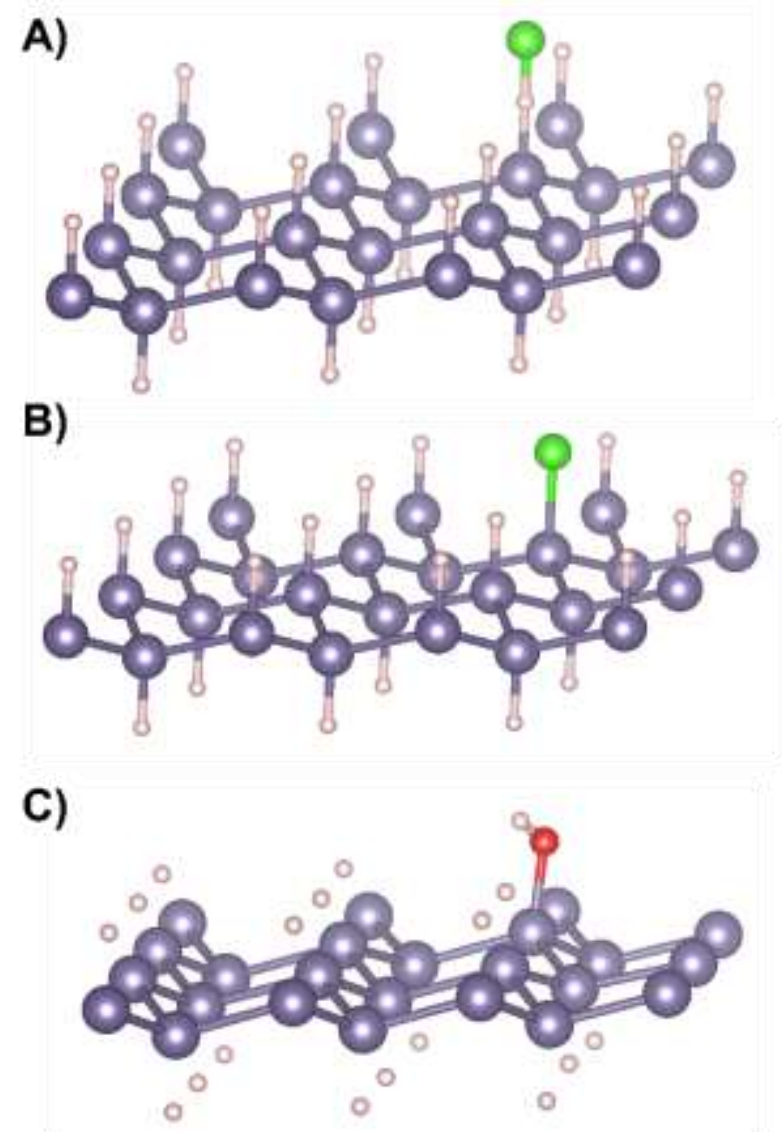

Figure S6. Ge-H with the relaxed geometries of $\mathrm{Cl}$ and $\mathrm{O}$ impurities in different configurations, (A) adsorbed Cl, (B) substituted $\mathrm{Cl}$, and (C) adsorbed -OH. Atom color scheme: Ge (purple), $\mathrm{H}$ (white), $\mathrm{Cl}$ (green), and $\mathrm{O}$ (red).

\section{References}

(1) Bianco, E.; Butler, S.; Jiang, S.; Restrepo, O. D.; Windl, W.; Goldberger, J. E. Stability and Exfoliation of Germanane: A Germanium Graphane Analogue. ACS Nano 2013, 7, 4414 4421. https://doi.org/10.1021/nn4009406.

(2) Rivillon, S.; Chabal, Y. J.; Amy, F.; Kahn, A. Hydrogen Passivation of Germanium (100) Surface Using Wet Chemical Preparation. Appl. Phys. Lett. 2005, 87, 253101. https://doi.org/10.1063/1.2142084. 